Владислав Д. Калашников, Алексей Ю. Егоров, Армен В. Согоян,

Анастасия В. Уланова, Андрей Б. Каракозов, Павел А. Баламутов

ВЛИЯНИЕ ПОВЫШЕННОЙ ИНТЕНСИВНОСТИ НАБОРА ДОЗЫ ИОНИЗИРУЮЩЕГО

ИЗЛУЧЕНИЯ НА СТОЙКОСТЬ КМОП МИКРОСХЕМ

СИСТЕМ ПЕРЕДАЧИ ИНФОРМАЦИИ

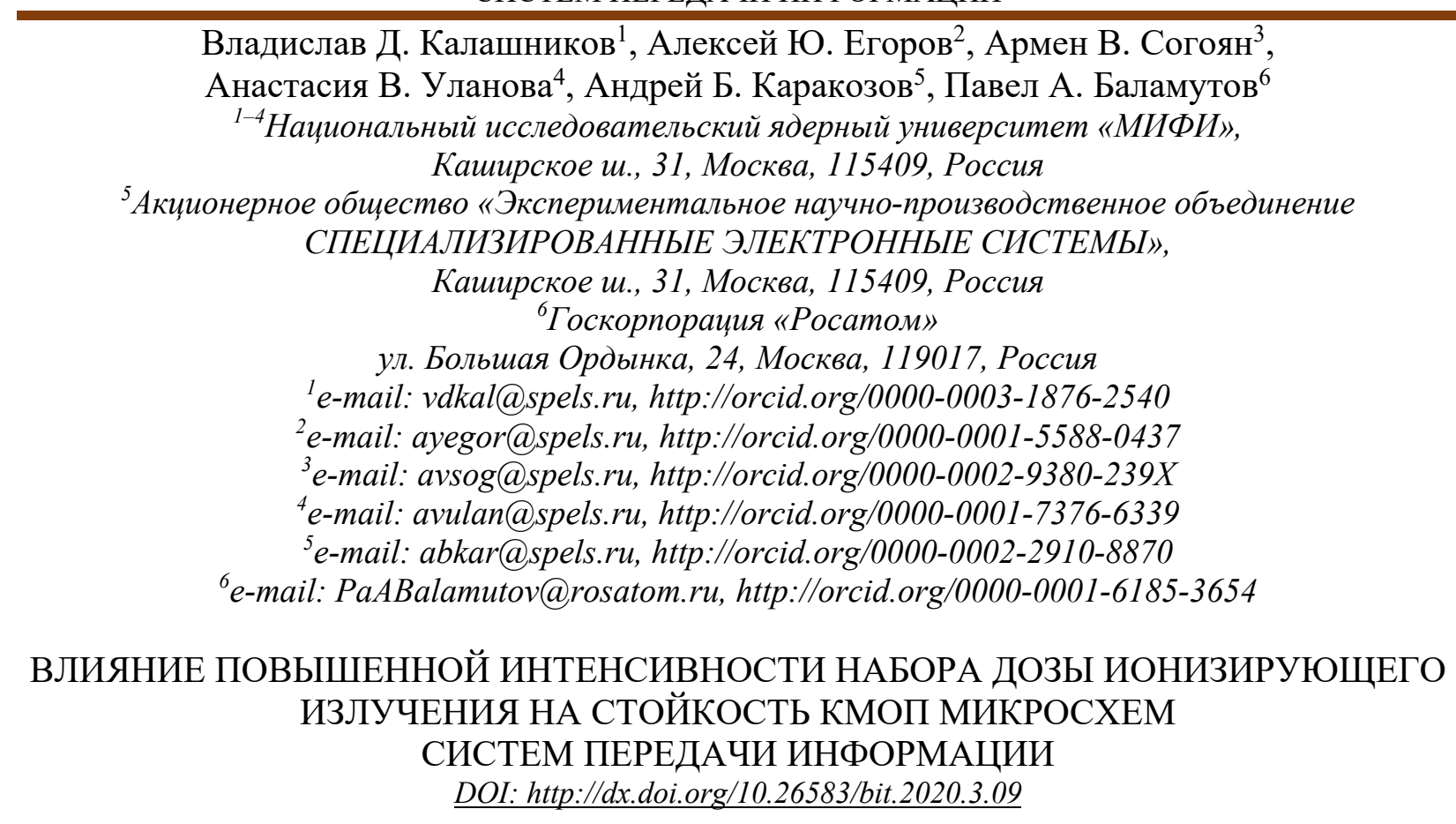

Аннотация. В данной работе проведено исследование влияния интенсивности воздействия при наборе дозы на стойкость КМОП интегральных схем (ИС). Исследовано 3 типа микросхем, изготовленных по проектным нормам от 0,8 мкм до 3 мкм, использующихся в различных системах передачи информации: 1586ИН4, НЕF4093ВТ и МС14504В. Исследования проводились с использованием ускорителя электронов У-31/33, работающего в тормозном режиме (средняя интенсивность воздействия) и режиме генерации пачки электронных импульсов (повышенная интенсивность). В результате исследований установлено, что повышение интенсивности воздействия на четыре порядка может приводить к снижению уровня стойкости ИС до 30 раз. Данное обстоятельство необходимо учитывать при проведении испытаний КМОП ИС на стойкость к воздействию импульсного ионизирующего излучения. Результаты, полученные в представленной работе, подтверждают теоретические представления о влиянии эффектов повышенной интенсивности набора дозы ионизирующего излучения на дозовую стойкость КМОП ИС.

Ключевые слова: безопасность информации, радиаџионная стойкость, КМОП СБИС, поглощённая доза, интенсивность набора дозы.

Для иитирования: КАЛАШНИКОВ, Владислав Д. et al. ВЛИЯНИЕ ПОВЫШЕННОЙ ИНТЕНСИВНОСТИ НАБОРА ДОЗЫ ИОНИЗИРУЮЩЕГО ИЗЛУЧЕНИЯ НА СТОЙКОСТЬ КМОП МИКРОСХЕМ СИСТЕМ ПЕРЕДАЧИ ИНФОРМАЦИИ. Безопасность информаиионных технологий, [S.l.], v. 27, n. 3, p. 98-103, 2020. ISSN 2074-7136. Доступно на: <https://bit.mephi.ru/index.php/bit/article/view/1296>. Дата docmyna: 14 sep. 2020. DOI: http://dx.doi.org/10.26583/bit.2020.3.09.

Vladislav D. Kalashnikov ${ }^{1}$, Alexey Yu. Egorov², Armen V. Sogoyan ${ }^{3}$,

Anastasia V. Ulanova ${ }^{4}$, Andrey B. Karakozov ${ }^{5}$, Pavel A. Balamutov 6

${ }^{1-4}$ National Research Nuclear University MEPhI (Moscow Engineering Physics Institute), Kashirskoe sh., 31, Moscow, 115409, Russia

${ }^{5}$ Joint Stock Company "Experimental Research and Production Association SPECIAL ELECTRONIC SYSTEM"

Kashirskoe sh., 31, Moscow, 115409, Russia

${ }^{6}$ State Atomic Energy Corporation «Rosatom»

Bolshaya Ordynka St., 24, Moscow, 119017, Russia

le-mail:vdkal@spels.ru,http://orcid.org/0000-0003-1876-2540

2e-mail: ayegor@spels.ru,http://orcid.org/0000-0001-5588-0437

3e-mail:avsog@spels.ru,http://orcid.org/0000-0002-9380-239X

4e-mail: avulan@spels.ru,http://orcid.org/0000-0001-7376-6339 
Владислав Д. Калашников, Алексей Ю. Егоров, Армен В. Согоян,

Анастасия В. Уланова, Андрей Б. Каракозов, Павел А. Баламутов

ВЛИЯНИЕ ПОВЫШЕННОЙ ИНТЕНСИВНОСТИ НАБОРА ДОЗЫ ИОНИЗИРУЮЩЕГО

ИЗЛУЧЕНИЯ НА СТОЙКОСТЬ КМОП МИКРОСХЕМ

СИСТЕМ ПЕРЕДАЧИ ИНФОРМАЦИИ

5e-mail:abkar@spels.ru,http://orcid.org/0000-0002-2910-8870

${ }^{6}$ e-mail: PaABalamutov@rosatom.ru,http://orcid.org/0000-0001-6185-3654

\title{
The influence of high ionizing dose rate on CMOS IC radiation hardness used in data transmission elements \\ DOI: http://dx.doi.org/10.26583/bit.2020.3.09
}

\begin{abstract}
In this work, the effect of the irradiation intensity on the radiation hardness of CMOS integrated circuits (ICs) was studied. We investigated 3 types of ICs manufactured according to design rules from 0.8 to 3 microns, and used in various information transmission systems: 1586IN4, HEF4093BT and MC14504B. The U-31/33 accelerator of electrons has been used for the study in both gamma-ray mode (for moderate intensity) and pulse electron generation mode (for high intensity). As a result, it was revealed that an increase in the intensity of exposure by 4 orders of magnitude might result in a radiation hardness decrease up to factor of 30 . This circumstance has to be taken into account when testing CMOS ICs for radiation hardness to high intensity effects. The obtained experimental data confirm theoretical understanding of the influence of increased irradiation intensity on the dose hardness of CMOS ICs.

Keywords: information security, radiation hardness, CMOS VLSI, total dose effect, dose-rate.

For citation: KALASHNIKOV, Vladislav D. et al. The influence of high ionizing dose rate on CMOS IC radiation hardness used in da-ta transmission elements. IT Security (Russia), [S.l.], v. 27, n. 3, p. 98-103, 2020. ISSN 20747136. Available at: <https://bit.mephi.ru/index.php/bit/article/view/1296>. Date accessed: 14 sep. 2020. DOI: http://dx.doi.org/10.26583/bit.2020.3.09.
\end{abstract}

\section{Введение}

Работоспособность накопителей памяти и обслуживающих их электронных компонентов (повторители, преобразователи уровня, приемопередатчики) зависит от внешних условий их эксплуатации. Одним из наиболее критичных внешних дестабилизирующих факторов, способных нарушить работоспособность устройств накопления и передачи информации является ионизирующее излучение (ИИ).

\section{1. Теоретические представления о повышенной интенсивности набора дозы ИИ}

Реакция современных КМОП СБИС на воздействие импульсного ИИ [1-3], в общем случае, будет определяться как процессами переноса неравновесного заряда в полупроводниковой структуре (эффекты «мощности дозы» - кратковременные параметрические и функциональные отказы, защелкивание), так и процессами переноса, захвата и релаксации радиационно-индуцированного заряда в паразитных диэлектрических структурах (эффекты «полной дозы») [4-10].

Сложившаяся практика радиационных испытаний изделий электронной компонентной базы (ЭКБ) предполагает определение времени потери работоспособности изделий по эффектам мощности дозы, тогда как переходным процессам, обусловленным накопленной дозой (перенос и релаксация заряда в диэлектрике) $[11,12]$, традиционно уделяется меньшее внимание. При этом экспериментальная оценка стойкости СБИС к воздействию импульсного ИИ по отношению к эффектам поглощенной дозы проводится на моделирующих установках при интенсивностях ИИ 10..1000 рад/с. Такой подход может приводить к существенной переоценке уровня стойкости и недооценке времени потери работоспособности СБИС по сравнению с воздействием импульсного излучения в реальных условиях [13]. Это обстоятельство требует развития методов испытаний, позволяющих учесть влияние эффектов переноса и релаксации заряда в диэлектрике при импульсном наборе дозы.

Как правило, на малых временах реакция изделия, связанная с формированием заряда в диэлектрике, определяется переносом заряда, а на более позднем этапе - его релаксацией (отжигом) $[14,15]$. Процесс релаксации захваченного заряда проявляется в 
зависимости уровня отказа изделия от интенсивности ИИ $[4,13]$ при временах облучения, превосходящих характерную длительность переноса носителей через диэлектрик.

\section{2. Объект исследования}

В работе исследовалось влияние данного механизма на уровень стойкости КМОП БИС при воздействии ИИ высокой интенсивности. Объектами исследований являлись микросхемы передачи информации [11], такие как: микросхемы серии 1586ИН4 (двухканальный приемник двуполярного последовательного кода), предназначенные для построения приемных устройств каналов информационного обмена по ГОСТ 18977 , изготовленные по КМОП технологии с проектными нормами 2 мкм; микросхемы HEF4093BT (четыре двухвходовых вентиля И-НЕ с входами триггера Шмидта), изготовленные по КМОП технологии, фирмы Phillips и микросхемы MC14504B (не инвертирующий ТТЛ-КМОП/КМОП-КМОП преобразователь логического уровня), изготовленные по КМОП технологии с проектными нормами 3 мкм с питаниями входа и выхода 3-18 В фирмы On Semiconductor.

На вход D1A микросхемы серии 1586ИН4 подавался периодический сигнал (меандр) частотой 1 МГц с размахом сигнала от $0 \mathrm{~B}$ до $+5 \mathrm{~B}$. На вход микросхемы D1B синфазно с входом D1A подавался периодический сигнал (меандр) частотой 1 МГц с размахом сигнала от 0 В до +5 В. На остальные входы микросхемы были поданы сигналы низкого логического уровня. Контроль функционирования производился с помощью осциллографа по выходному сигналу микросхемы OUTA_1. Критерием работоспособности являлось наличие на выходе переменного сигнала (меандра) с амплитудой не менее 1,65 В.

На вход I1 микросхемы НЕF4093ВТ подавался периодический сигнал (меандр) частотой 20 кГц. Остальные входы микросхемы были подтянуты к питанию с помощью резисторов номиналом 5 кОм. Контроль функционирования проводился с помощью осциллографа по выходному сигналу О1 микросхемы. Критерием работоспособности являлось наличие на выходе переменного сигнала (меандра).

На входы А⿰氵 микросхемы МC14504В подавался периодический сигнал (меандр) частотой 1 МГц с размахом сигнала от 0 В до $+3,6$ В. На остальные входы микросхемы были поданы сигналы низкого логического уровня. Контроль функционирования проводился с помощью осциллографа по выходным сигналам микросхем Аоuт. Критерием работоспособности являлось наличие на выходе переменного сигнала (меандра).

Исследования проводились с использованием ускорителя электронов «У-31/33», работающего в режиме тормозного излучения и в режиме электронного пучка. Образцы были разделены на две группы. Образцы первой группы облучались при интенсивностях 20 ед./с (1586ИН4), 40 ед./с (НЕF4093ВТ), и 134 ед./с (МС14504В), образцы второй группы

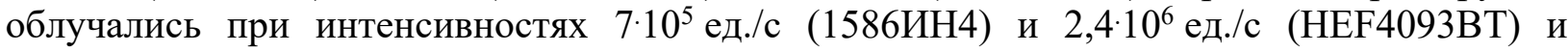
0,95.10 $10^{6}$ ед./с (МС14504В). Зависимости выходного уровня микросхемы 1586ИН4 от набранной дозы приведены на рис. 1. 
Владислав Д. Калашников, Алексей Ю. Егоров, Армен В. Согоян,

Анастасия В. Уланова, Андрей Б. Каракозов, Павел А. Баламутов

ВЛИЯНИЕ ПОВЫШЕННОЙ ИНТЕНСИВНОСТИ НАБОРА ДОЗЫ ИОНИЗИРУЮЩЕГО

ИЗЛУЧЕНИЯ НА СТОЙКОСТЬ КМОП МИКРОСХЕМ СИСТЕМ ПЕРЕДАЧИ ИНФОРМАЦИИ

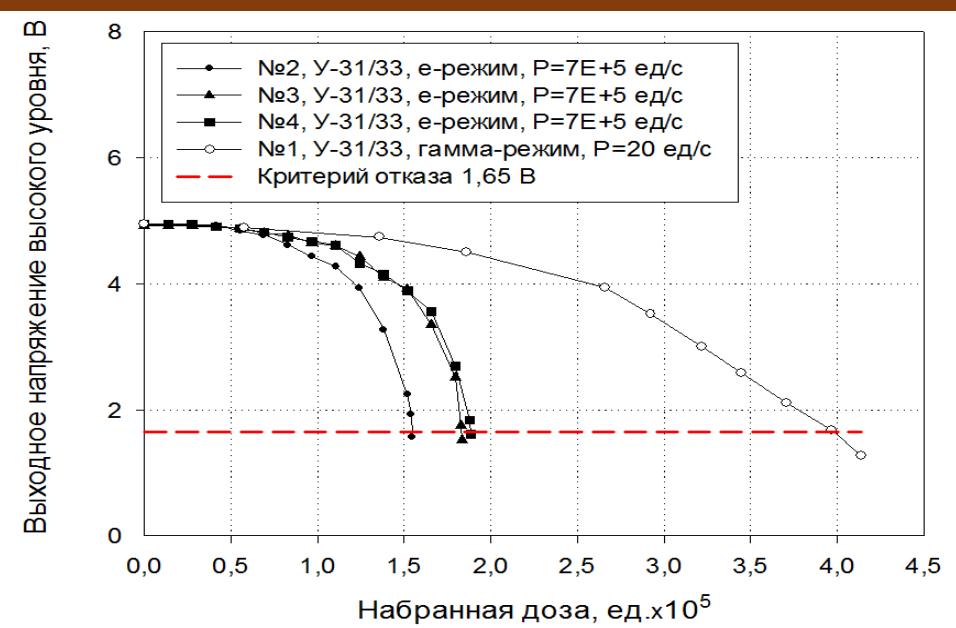

Рис. 1. Зависимости выходного напряжения высокого уровня $\mathrm{U}_{\mathrm{OH}}$ микросхемы 1586ИН4 от набранной дозы при использовании линейного ускорителя электронов «У-31/33», работающего в электронном (черные маркеры) и тормозном

(белые маркеры) режимах

Fig. 1. The output voltage VOH of the 1586IN4 IC as function of total dose for different irradiation intensities. Black markers - high intensity (pulse electron irradiation); white markers - moderate intensity (gamma ray irradiation).

\section{3. Результаты исследований}

Установленные уровни отказа микросхем приведены в табл. 1. Отличие в уровнях стойкости составило около трех раз при разнице в интенсивностях на четыре порядка (для 1586ИН4) и на пять порядков (для НЕF4093ВТ).

Таблица 1. Уровни отказа микросхем

\begin{tabular}{|c|c|c|}
\hline Образец & Интенсивность, ед/с & Доза отказа, ед. \\
\hline \multicolumn{3}{|c|}{ Микросхемы 1586ИН4 } \\
\hline 1 & 20 & $39 \cdot 10^{4}$ \\
\hline 2 & $7 \cdot 10^{5}$ & $15 \cdot 10^{4}$ \\
\hline 3 & $7 \cdot 10^{5}$ & $18 \cdot 10^{4}$ \\
\hline 4 & $7 \cdot 10^{5}$ & $19 \cdot 10^{4}$ \\
\hline \multicolumn{3}{|c|}{ Микросхемы HEF4093BT } \\
\hline 21 & 40 & $4,3 \cdot 10^{4}$ \\
\hline 25 & 40 & $4,7 \cdot 10^{4}$ \\
\hline 2 & $2,4 \cdot 10^{6}$ & $1,7 \cdot 10^{4}$ \\
\hline 8 & $2,4 \cdot 10^{6}$ & $1,7 \cdot 10^{4}$ \\
\hline 3 & $2,4 \cdot 10^{6}$ & $1,7 \cdot 10^{4}$ \\
\hline \multicolumn{3}{|c|}{ Микросхемы МС14504B } \\
\hline 4 & $0,95 \cdot 10^{6}$ & $1 \cdot 10^{4}$ \\
\hline 5 & $0,95 \cdot 10^{6}$ & $1 \cdot 10^{4}$ \\
\hline 1 & 134 & $31 \cdot 10^{4}$ \\
\hline 2 & 134 & $29 \cdot 10^{4}$ \\
\hline
\end{tabular}




\section{Заключение}

Полученные в работе данные дают количественную оценку влияния высокой интенсивности воздействия на дозовую стойкость исследованных объектов. Так, для микросхемы МС14504В соотношение уровней отказа при средней и высокой интенсивностях составляет около 30 раз, а для остальных типов - 3-4 раза. Результаты экспериментов позволяют сделать вывод о значимости исследованного эффекта.

\section{СПИСОК ЛИТЕРАТУРЫ:}

1. Никифоров А.Ю. Радиационные эффекты в КМОП ИС // Телец В.А., Чумаков А.И. - М: Радио и связь, 1994. - $165 \mathrm{c}$.

2. Устюжанинов В.Н. Эффекты импульсного облучения цифровых электронных систем // Вопросы атомной науки и техники. Серия: Физика радиационного воздействия на радиоэлектронную аппаратуру. 2013. № 1. C 50-54. URL: https://elibrary.ru/title_about.asp?id=25748 (дата обращения: 01.08.2020).

3. Устюжанинов В.Н. Ионизационные эффекты импульсного облучения БИС // Вопросы атомной науки и техники. Серия: Физика радиационного воздействия на радиоэлектронную аппаратуру. 2013. № 1. C. 55-60.

4. Ionizing Radiation Effects in MOS devices and Circuits, ed. by T.P.Ma and P.V. Dressendorfer, J.Wiley\& Sons, New York, 1989. DOI: https://doi.org/10.1148/radiology.174.3.886.

5. H. Barnaby. Total-ionizing-dose effects in modern CMOS technologies, Nuclear Science, IEEE Transactions on Vol. 53. P. 3103-3121, Dec 2006. DOI: https://doi.org/10.1109/TNS.2006.885952.

6. Согоян А.В. Оценка стойкости КМОП СБИС к фактору поглощенной дозы при воздействии импульсного излучения // Микроэлектроника. 2011. Т. 40, № 3. С. 200-208 URL: https://www.elibrary.ru/item.asp?id=15257433 (дата обращения: 01.08.2020).

7. Согоян А.В., Давыдов Г.Г. Особенности формирования и релаксации заряда в КНС структурах при воздействии ионизирующего излучения // Микроэлектроника. 2011. Т. 40, № 3. С. 209-223 URL: https://elibrary.ru/title_about.asp?id=7900 (дата обращения: 01.08.2020).

8. H. Barnaby, M. Mclain, I. S. Esqueda. Total-ionizing-dose effects on isolation oxides in modern CMOS technologies, Nuclear Instruments and Methods in Physics Research B 261 (2007) 1142-1145. DOI: https://doi.org/ 10.1016/j.nimb.2007.03.109.

9. Y.M. Moskovskaya, A. Y. Nikiforov, D.V.Bobrovsky, A.V. Ulanova, and A. A Zhukov. Process Parameters Variations Influence on CMOS IC's Hardness to total Ionizing Dose, in Proc. 30th Int. Conf. on Microelectronics, $\begin{array}{llllll}\text { MIEL 2017; } & \text { Nis, Serbia, October } 2017 . & \text { P. }\end{array}$ DOI: https://doi.org/10.1109/MIEL.2017.8190120.

10. Барбашов, Вячеслав М.; Калашников, Олег А. Функционально-логическое моделирование дозовых радиационных отказов сф-блоков систем на кристалле. Безопасность информационных технологий,

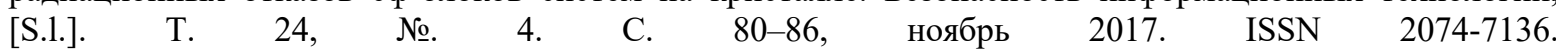
URL: $\quad<$ https://bit.mephi.ru/index.php/bit/article/view/283> (дата обращения: $\quad 01.08 .2020$ ). DOI: https://doi.org/10.26583/bit.2017.4.09.

11. Киргизова А.В., Никифоров А.Ю., Григорьев Н.Г., Поляков И.В., Скоробогатов П.К. Доминирующие механизмы информационных сбоев КМОП КНС БИС оперативных запоминающих устройств при воздействии импульсного ионизирующего излучения» // Микроэлектроника . 2006, Т. 35, № 3. C. $191-208$.

12. Зинченко В.Ф., Романенко А.А., Усеинов Р.Г., Ужегов В.М. Радиационные эффекты в изделиях полупроводниковой микроэлектроники современных технологий, Вопросы атомной науки и техники. Серия: Физика радиационного воздействия на радиоэлектронную аппаратуру. 2016. № 1. С 29-36. URL: https://www.elibrary.ru/item.asp?id=26131819 (дата обращения: 01.08.2020).

13. Никифоров А.Ю., Согоян А.В. Моделирование дозовых эффектов в паразитных МОП-структурах КМОП БИС при воздействии высокоинтенсивного импульсного ионизирующего излучения // Микроэлектроника. 2004. Т. 33. № 2. C. 108. URL: https://www.elibrary.ru/item.asp?id=17662355 (дата обращения: 01.08.2020).

14. Sogoyan A.V. and Polunin V.A. Modeling of recombination in $\mathrm{SiO}_{2}$ under the effect of ionizing radiation by the Monte Carlo method, Russian Microelectronics. Vol. 40, no. 3. P. 176-184, 2011. DOI: https://doi.org/10.1134/S1063739711030061.

15. Таперо К.И. Эффекты низкоинтенсивного облучения в приборах и интегральных схемах на базе кремения // Известия высших учебных заведений. Материалы электронной техники. 2016. Т. 19, № 1. C. 5-21. DOI: https://doi.org/10.17073/1609-3577-2016-1-5-21. 
Владислав Д. Калашников, Алексей Ю. Егоров, Армен В. Согоян,

Анастасия В. Уланова, Андрей Б. Каракозов, Павел А. Баламутов

\section{ВЛИЯНИЕ ПОВЫШЕННОЙ ИНТЕНСИВНОСТИ НАБОРА ДОЗЫ ИОНИЗИРУЮЩЕГО ИЗЛУЧЕНИЯ НА СТОЙКОСТЬ КМОП МИКРОСХЕМ СИСТЕМ ПЕРЕДАЧИ ИНФОРМАЦИИ}

REFERENCES:

[1] Nikiforov A.Yu. Radiation effects in CMOS IC. Telets V.A., Chumakov A.I. - M.: Radio and communications, 1994. - 165 p. (in Russian).

[2] Ustyuzhaninov V.N. Effekty impul'snogo oblucheniya cifrovyh elektronnyh sistem. Voprosy atomnoj nauki i tekhniki. Seriya: Fizika radiacionnogo vozdejstviya na radioelektronnuyu apparaturu. 2013. No 1. S. 50-54. URL: https://elibrary.ru/title_about.asp?id=25748 (accessed: 01.08.2020) (in Russian).

[3] Ustyuzhaninov V.N. Ionizacionnye effekty impul'snogo oblucheniya BIS. Voprosy atomnoj nauki i tekhniki. Seriya: Fizika radiacionnogo vozdejstviya na radioelektronnuyu apparaturu. 2013. No 1. S. 55-60 (in Russian).

[4] Ionizing Radiation Effects in MOS devices and Circuits, ed. by T.P.Ma and P.V. Dressendorfer, J.Wiley\& Sons, New York, 1989. DOI: https://doi.org/10.1148/radiology.174.3.886.

[5] H. Barnaby. Total-ionizing-dose effects in modern CMOS technologies, Nuclear Science, IEEE Transactions on, Vol. 53. P. 3103-3121, Dec 2006 DOI: https://doi.org/10.1109/TNS.2006.885952.

[6] Sogoyan A.V. Otsenka stojkosti KMOP SBIS k faktoru pogloshchennoj dozy pri vozdejstvii impul'snogo izlucheniya. Mikroelektronika. 2011. T. 40, no 3. S. 200-208 (in Russian). URL: https://www.elibrary.ru/item.asp?id=15257433 (accessed: 01.08.2020).

[7] Sogoyan A.V., Davydov G.G. Osobennosti formirovaniya i relaksacii zaryada v KNS strukturah pri vozdejstvii ioniziruyushchego izlucheniya. Mikroelektronika. 2011. T. 40, no 3. S. $209-223$. URL: https://elibrary.ru/title_about.asp?id=7900 (accessed: 01.08.2020) (in Russian).

[8] H. Barnaby, M. Mclain, I.S. Esqueda. Total-ionizing-dose effects on isolation oxides in modern CMOS technologies, Nuclear Instruments and Methods in Physics Research B 261 (2007), 1142-1145. DOI: https://doi.org/10.1016/j.nimb.2007.03.109.

[9] Y.M. Moskovskaya, A.Y. Nikiforov, D.V.Bobrovsky, A.V. Ulanova and A.A Zhukov. Process Parameters Variations Influence on CMOS IC's Hardness to total Ionizing Dose, in Proc. 30th Int. Conf. on Microelectronics, MIEL 2017; Nis, Serbia, October 2017. P. 275-277.

[10] Barbashov, Vyacheslav M.; Kalashnikov, Oleg A. Functional-logic simulation of IP-blocks dose functional failures. IT Security (Russia), [S.1.], V. 24, no 4. P. 80-86, nov. 2017. ISSN 2074-7136. URL: $\quad<$ https://bit.mephi.ru/index.php/bit/article/view/283>. Date accessed: $10 \quad$ sep. 2020. DOI: http://dx.doi.org/10.26583/bit.2017.4.09.

[11] Kirgizova A.V., Nikiforov A.Yu., Grigor'ev N.G., Polyakov I.V., Skorobogatov P.K. Dominiruyushchie mekhanizmy informacionnyh sboev KMOP KNS BIS operativnyh zapominayushchih ustrojstv pri vozdejstvii impul'snogo ioniziruyushchego izlucheniya. Mikroelektronika - 2006. T. 35, no 3. S. 191-208 (in Russian).

[12] Zinchenko V.F., Romanenko A.A., Useinov R.G., Uzhegov V.M. Radiacionnye effekty v izdeliyah poluprovodnikovoj mikroelektroniki sovremennyh tekhnologij, Voprosy atomnoj nauki i tekhniki. Seriya: Fizika radiacionnogo vozdejstviya na radioelektronnuyu apparaturu. 2016. no $1 . \quad$ S. $29-36$. URL: https://www.elibrary.ru/item.asp?id=26131819 (accessed: 01.08.2020) (in Russian).

[13] Nikiforov A.Yu., Sogoyan A.V. Modelirovanie dozovyh effektov v parazitnyh MOP-strukturah KMOP BIS pri vozdejstvii vysokointensivnogo impul'snogo ioniziruyushchego izlucheniya. Mikroelektronika. 2004. T. 33, no 2. S. 108. URL: https://www.elibrary.ru/item.asp?id=17662355 (in Russian).

[14] Sogoyan A.V. and Polunin V.A. Modeling of recombination in $\mathrm{SiO}_{2}$ under the effect of ionizing radiation by the Monte Carlo method, Russian Microelectronics. Vol. 40, no. 3. P. 176-184, 2011. DOI: https://doi.org/10.1134/S1063739711030061.

[15] K.I. Tapero. Effekty nizkointensivnogo oblucheniya v priborah i integral'nyh skhemah na baze kremeniya. Izvestiya vysshih uchebnyh zavedenij. Materialy elektronnoj tekhniki. 2016. T. 19, no 1. S 5-21. DOI: https://doi.org/10.17073/1609-3577-2016-1-5-21 (in Russian).

Поступила в редакиию - 26 июля 2020 г. Окончательный вариант - 26 августа 2020 г. Received - July 26, 2020. The final version-August 26, 2020. 